\title{
Erratum to: Impact of an integrated obesity management system on patient's care - research protocol
}

Jean-Patrice Baillargeon ${ }^{1}$, Denise St-Cyr-Tribble ${ }^{2}$, Marianne Xhignesse ${ }^{3}$, Andrew Grant ${ }^{4 \dagger}$, Christine Brown ${ }^{1 \dagger}$, Thomas G. Poder ${ }^{5}$ and Marie-France Langlois ${ }^{1 *}$

After publication of the original article [1] the authors requested that the author list be updated. Therefore, we would like to acknowledge Thomas G. Poder as an author of this manuscript. The order of the author list has also been changed. The original order appeared as follows:

Jean-Patrice Baillargeon ${ }^{1}$, Denise St-Cyr-Tribble ${ }^{2+}$, Marianne Xhignesse ${ }^{3 \dagger}$, Andrew Grant $^{4 \dagger}$, Christine Brown $^{1+}$ and Marie-France Langlois ${ }^{1 *}$

The new order, however, should be as listed below:

Jean-Patrice Baillargeon ${ }^{1}$, Denise St-Cyr-Tribble ${ }^{2+}$, Marianne Xhignesse ${ }^{3+}$, Andrew $\mathrm{Grant}^{4+}$, Christine Brown $^{1+}$, Thomas G. Poder ${ }^{5}$ and Marie-France Langlois ${ }^{1 *}$

\begin{abstract}
Author details
'Division of Endocrinology, Department of Medicine, Université de Sherbrooke, Sherbrooke, Québec, Canada. ${ }^{2}$ École des sciences infirmières, Université de Sherbrooke, Sherbrooke, Québec, Canada. ${ }^{3}$ Department of Family and Emergency Medicine, Université de Sherbrooke, Sherbrooke, Québec, Canada. ${ }^{4}$ Department of Biochemistry, Université de Sherbrooke, Sherbrooke, Québec, Canada. ${ }^{5}$ CRCHUS and UETMIS, CIUSSS de l'Estrie CHUS, Sherbrooke, Québec, Canada.
\end{abstract}

Received: 15 April 2016 Accepted: 15 April 2016

Published online: 29 April 2016

\section{Reference}

1. Baillargeon JP, et al. Impact of an integrated obesity management system on patient's care - research protocol. BMC Obesity. 2014;1:19.

\footnotetext{
* Correspondence: marie-france.langlois@usherbrooke.ca

${ }^{\dagger}$ Equal contributors

'Division of Endocrinology, Department of Medicine, Université de Sherbrooke, Sherbrooke, Québec, Canada
}

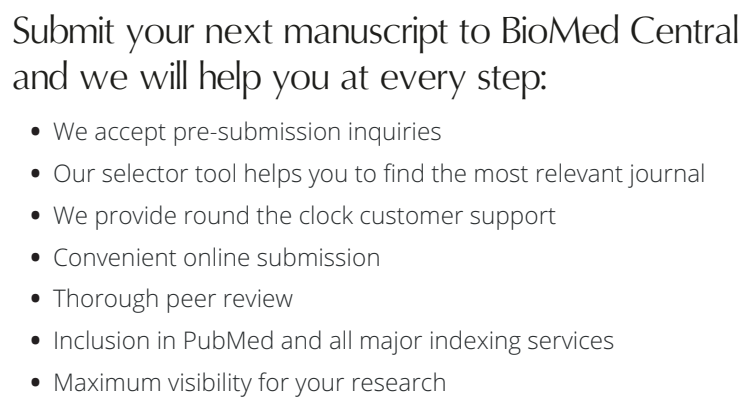
and we will help you at every step:

- We accept pre-submission inquiries

- Our selector tool helps you to find the most relevant journal

- We provide round the clock customer support

- Convenient online submission

- Thorough peer review

- Inclusion in PubMed and all major indexing services

- Maximum visibility for your research

Submit your manuscript at www.biomedcentral.com/submit 